Please do not remove this page

RMIT

UNIVERSITY

\title{
Transparent amorphous strontium titanate resistive memories with transient photo-response
}

Ahmed, Taimur; Walia, Sumeet; Kim, Jeeson; Nili Ahmadabadi, Hussein; Ramanathan, Rajesh; Mayes, Edwin; Lau, Desmond

https://researchrepository.rmit.edu.au/esploro/outputs/9921863204601341/filesAndLinks?institution=61RMIT_INST\&index=null

Ahmed, T., Walia, S., Kim, J., Nili Ahmadabadi, H., Ramanathan, R., Mayes, E., Lau, D., Kavehei, O., Bansal, V., Bhaskaran, M., \& Sriram, S. (2017). Transparent amorphous strontium titanate resistive memories with transient photo-response. Nanoscale, 9(38), 14690-14702. https://doi.org/10.1039/c7nr04372d Document Version: Accepted Manuscript

Published Version: https://doi.org/10.1039/c7nr04372d

Repository homepage: https://researchrepository.rmit.edu.au

This journal is (c) The Royal Society of Chemistry 2017

Downloaded On 2023/04/26 17:40:25 +1000 
Thank you for downloading this document from the RMIT Research Repository.

The RMIT Research Repository is an open access database showcasing the research outputs of RMIT University researchers.

RMIT Research Repository: http://researchbank.rmit.edu.au/

\section{Citation:}

Ahmed, T, Walia, S, Kim, J, Nili Ahmadabadi, H, Ramanathan, R, Mayes, E, Lau, D, Kavehei, O, Bansal, V, Bhaskaran, M and Sriram, S 2017, 'Transparent amorphous strontium titanate resistive memories with transient photo-response', Nanoscale, vol. 9, no. 38, pp. 14690-14702.

See this record in the RMIT Research Repository at:

https://researchbank.rmit.edu.au/view/rmit:45811

Version: Accepted Manuscript

Copyright Statement:

(C) The Royal Society of Chemistry 2017

\section{Link to Published Version:}

https://dx.doi.org/10.1039/c7nr04372d 


\section{Nanoscale}

\section{ARTICLE}

\section{Transparent Amorphous Strontium Titanate Resistive Memories with Transient Photo-Response}

Received 00th January 20xx, Accepted 00th January 20xx

DOI: $10.1039 / x 0 x \times 00000 x$

www.rsc.org/

\author{
Taimur Ahmed, ${ }^{a}$ Sumeet Walia, ${ }^{a}$ Jeeson Kim, ${ }^{a}$ Hussein Nili, ${ }^{a, b}$ Rajesh Ramanathan, ${ }^{c}$ \\ Edwin L. H. Mayes, ${ }^{d}$ Desmond W. M. Lau, ${ }^{\mathrm{e}}$ Omid Kavehei, ${ }^{a}$ Vipul Bansal, ${ }^{\mathrm{c}}$ Madhu Bhaskaran ${ }^{\mathrm{a}}$ and \\ Sharath Sriram ${ }^{\mathrm{a}}$
}

\section{Introduction}

Transparent electronics is of increasing interest for the next generation of smart electronic circuitry that can enable devices capable of displaying information on surfaces such as glass windows. For a fully transparent integrated circuit, high transparency is also desirable in its important circuit element: the memory unit. For optical transparency without compromising memory density, inherent transparency is needed in a single memory unit. That raises the requirement of both the active switching material and the electrodes being transparent. In the past, a variety of materials and microstructures including metal oxides, ${ }^{1-3}$ nanotubes/rods ${ }^{4,5}$ and graphene based hybrid structures ${ }^{6-8}$ have been studied separately as either opto-tunable or transparent memory systems. However, it is still challenging to achieve

\footnotetext{
Functional Materials and Microsystems Research Group and Micro Nano Research Facility, RMIT University, Melbourne, Australia. E-mail: taimur.ahmed@rmit.edu.au, sharath.sriram@rmit.edu.au

. Electrical and Computer Engineering Department, University of California Santa Barbara, Santa Barbara, CA 93106 (USA).

Ian Potter NanoBioSensing Facility, NanoBiotechnology Research Laboratory, School of Science, RMIT University, Melbourne, VIC 3001, Australia.

d. RMIT Microscopy and Microanalysis Facility, RMIT University, Melbourne, VIC 3001, Australia.

e. ARC Centre of Excellence for Nanoscale BioPhotonics \& School of Science, RMIT University, Melbourne, VIC 3001, Australia.

Electronic Supplementary Information (ESI) available: [details of any supplementary information available should be included here]. See DOI: $10.1039 / x 0 x x 00000 x$
}

optical/electrical tunability and transparency on a single memory cell that is capable of high density data storage.

As the mechanisms governing many resistive switching systems are not yet fully understood and well-established, they are still part of ongoing research. Thus, assessment of any potential application in transparent electronics depends on the actual demonstration of a working system. Here, we report fully transparent resistive random-access memories ( $t$ ReRAMs) that feature high transparency (>85\% including the substrate). Amorphous $\mathrm{SrTiO}_{3}$ (a-STO) is chosen as the functional oxide owing to its ability to harbor oxygen vacancies $\left(\mathrm{V}_{\mathrm{o}} \mathrm{s}\right)$ in the lattice, which can be easily tailored. ${ }^{9-11}$ Our $t$ ReRAM cells are implemented in a stacked configuration with a bilayer structure comprising two a-STO layers with varying concentrations of $\mathrm{V}_{\mathrm{o}} \mathrm{s}$. It has been previously reported that a bilayer structure with each layer having different stoichiometry exhibits better switching performances compared to a single layer. ${ }^{12-15}$ This is mainly because a combination of two layers, each with an intrinsic Schottky barrier, eliminates the need for using an additional element (generally a transistor or diode) to remove stray leakage current paths in high-density memory arrays. ${ }^{13}$ Indium tin oxide (ITO), a well-known transparent conductor is used as the top and bottom electrode. The entire system is fabricated on glass, rendering all constituent elements of the memory unit to be optically transparent.

The $t$-ReRAMs show excellent performance with switching ratios over 2 orders of magnitude and retention over $10^{5} \mathrm{~s}$. 
Furthermore, the memory cells also show a transient photoresponse in their high resistive state (HRS) under UV and blue light excitation. The switching mechanism is investigated using area dependent electrical characterization and PL mapping of devices in different resistive states. It is established that the switching in our devices arises due to the electroforming induced filaments in the amorphous oxide.

\section{Experimental}

\section{Material deposition and device fabrication}

The $t$-ReRAMs are fabricated in a cross-point configuration on a glass (Pyrex) substrate via standard photolithography/lift-off and sputter deposition processes. To study the effect of cell size on the resistive switching characteristics, different $t$-ReRAM cells are fabricated with lateral dimensions of $2 \times 2 \mu \mathrm{m}^{2}, 4 \times 4 \mu \mathrm{m}^{2}, 10 \times 10 \mu \mathrm{m}^{2}$, $20 \times 20 \mu \mathrm{m}^{2}, \quad 40 \times 40 \mu \mathrm{m}^{2}, \quad 80 \times 80 \mu \mathrm{m}^{2}$ and $100 \times 100 \mu \mathrm{m}^{2}$ (see Supporting Information, Fig. S1). Each $t$-ReRAM cell comprises of a bilayer homojunction of $a$-STO, with different oxygen vacancy concentrations, sandwiched between two transparent conductive oxide (TCO) electrodes. A pair of bottom $(30 \mathrm{~nm})$ and top $(40 \mathrm{~nm})$ ITO electrodes are RF sputtered in a pure $\mathrm{Ar}$ atmosphere from a commercial ITO source $\left(\mathrm{In}_{2} \mathrm{O}_{3}: \mathrm{SnO}_{2}\right.$ in a 90:10 wt\%, Testbourne Ltd.). A detailed description on the stoichiometry of sputtered ITO thin films is provided in Supporting Information (Fig. S2). Room temperature RF sputtering of ITO in pure Ar environment results in thin films with poor transparency (Supporting Information, Fig. S3). In order to enhance optical transparency, the bottom ITO electrodes are annealed in ambient conditions at $400{ }^{\circ} \mathrm{C}$ for one hour in a furnace. Subsequently, the bilayer homojunction of $a$-STO is $\mathrm{RF}$ sputtered at room temperature from a ceramic $\mathrm{SrTiO}_{3}$ source (99.95\% pure, Testbourne Ltd.). A detailed description of the room temperature synthesis of $a$-STO thin films with different stoichiometry is reported in our earlier studies. ${ }^{9,} 16$ In order to enable repeatable resistive switching in $t$-ReRAM cells, an underlying $\mathrm{Ti}(5 \mathrm{~nm})$ layer is DC sputtered in a pure Ar environment and capped with a top ITO electrode to form asymmetric top ITO/Ti/a-STO ${ }_{x}$ interface. Single layer and bilayer $t$-ReRAM cells with symmetric interfaces are also fabricated by following the processes explained above. Their electrical characterization shows unstable resistive switching behavior (Supporting Information, Fig. S4), necessitating the use of asymmetric interfaces in this work.

\section{X-ray photoelectron spectroscopy}

$\mathrm{X}$-ray photoelectron spectroscopy (XPS) analysis is conducted by using a Thermo Scientific K-Alpha instrument. An aluminium $K \alpha$ radiation source with energy of $1486.7 \mathrm{eV}$ is used. The core-level elemental spectra are collected from bare $a-\mathrm{STO}_{x}$ and $a-\mathrm{STO}_{y}$ thin films $(15 \mathrm{~nm}$ thick, without top ITO/Ti electrode) sputtered on $\mathrm{SiO}_{2} / \mathrm{Si}$ substrates using identical deposition conditions as used for $t$-ReRAMs. The elemental spectra are collected under high vacuum conditions $\left(<5 \times 10^{-}\right.$ ${ }^{8}$ Torr) by using the $\mathrm{X}$-ray beam of $400 \mu \mathrm{m}$ in diameter. The binding energies of all principal elements are referenced to the adventitious carbon binding energy (C 1s) of $285 \mathrm{eV}$.

\section{Electrical characterization}

The electrical measurements of the $t$-ReRAMs are performed using an Agilent 2912A source meter and a Keithley 4200SCS semiconductor characterization system for two-probe measurements.

\section{Photoelectric and optical characterization}

A commercial four wavelength $(365,455,530$, and $650 \mathrm{~nm}$ ) high power light-emitting diode (LED, Thorlabs, Inc.) is used as an illumination source. The illumination power is calibrated by a commercial UV-enhanced silicon photodetector (Newport Corporation). Optical transmission and reflectance of the fabricated $t$-ReRAM are measured by using the CRAIC 20/30 XL UV-Vis micro-spectrophotometer.

\section{Photoluminescence spectroscopy}

The photoluminescence ( $\mathrm{PL}$ ) emission spectra are obtained by using a Horiba Scientific FluoroMax-4 spectrofluorometer. All spectra are collected at room temperature, with $300 \mathrm{~nm}$ excitation wavelength, from the as-deposited bare $a$-STO thin films.

The PL mapping is carried out on a $4 \times 4 \mu \mathrm{m}^{2}$ Device BL15 in its virgin, high and low resistance states. The PL maps of the electroformed cell are obtained after subjecting to at least 20 switching cycles. A custom-built scanning confocal fluorescence microscope with a high numerical aperture (0.9) objective is used. A $405 \mathrm{~nm}$ laser as an excitation source combined with avalanche photo-diodes (APD, SPCM-AQRH-14) is employed for imaging and a spectrometer (Princeton Instruments, SpectraPro) with a PIXIS CCD camera to collect spectra. The lateral resolution is set to $300 \mathrm{~nm}$ with dwelling time of $1 \mathrm{~ms}$. During the PL mapping the cross-point $t$-ReRAM cells are photo-excited from the top surface.

\section{Transmission electron spectroscopy}

For transmission electron microscopy (TEM) analysis, samples are prepared by focused ion beam (FIB) cuts through the $t$ ReRAM cells using a FEI Scios DualBeam ${ }^{\mathrm{TM}}$ system. Crosssectional micrographs and electron energy loss spectroscopy (EELS) line scans are performed using a JEOL 2100F scanning transmission electron microscope (STEM) with attached Tridium Gatan image filter. The STEM micrographs and EELS spectra are collected using a $1.5 \mathrm{~nm}$ beam spot size. The oxygen $K$-edge intensity is used to determine the relative oxygen content across the $t$-ReRAM structure. The background is fitted using a power law fit and the influence of nearby peaks and plural scattering are reduced by a narrow signal window.

\section{Results and discussion}

\section{Optical transmission characterization}

A schematic of the fabricated cross-point $t$-ReRAM cell is shown in Fig. 1a, where the cell area (marked inside a rectangle on the cross-point structure) is magnified for a clear 
illustration of the multilayer cell structure. A homojunction of functional $a$-STO oxides with different concentration of $\mathrm{V}_{\mathrm{o}} \mathrm{S}$ is achieved by sputtering STO in different $\mathrm{O}_{2} / \mathrm{Ar}$ flow rates while keeping the deposition pressure constant at $3.5 \times 10^{-3}$ Torr. A thin $a$-STO layer, as a base layer (BL) of the stack, is sputtered in $5 \%$ of oxygen (i.e., $\mathrm{O}_{2}: \mathrm{Ar}: 5 \%: 95 \%$ ) on the bottom ITO electrode. We denote this base layer as $a-\mathrm{STO}_{y}$ for further discussions in this work. Following the $a$-STO $y$ sputtering, a second $a$-STO layer is sputtered (on $a$-STO $/$ ITO) in a pure $\mathrm{Ar}$ environment ( $0 \%$ oxygen), denoted by $a-\mathrm{STO}_{x}$ in this work. The stoichiometry of each $a$-STO layer is explained later in the manuscript. As a top electrode, a thin film of $\mathrm{Ti}(5 \mathrm{~nm})$ is DC sputtered in a pure Ar environment on the oxide homojunction and capped with an ITO $(40 \mathrm{~nm})$ electrode. The Ti layer is expected to act as a sink for oxygen ions that evolve during electric field induced redox reactions resulting in the partial oxidation of $\mathrm{Ti}$ layer to $\mathrm{Ti}_{2} \mathrm{O}_{3}$ at the top interface. The role of top $\mathrm{Ti} / \mathrm{Ti}_{2} \mathrm{O}_{3} / \mathrm{a}-\mathrm{STO}_{\mathrm{x}}$ interface in resistive switching, in our $\mathrm{t}$ ReRAM cells, is discussed in the following sections.

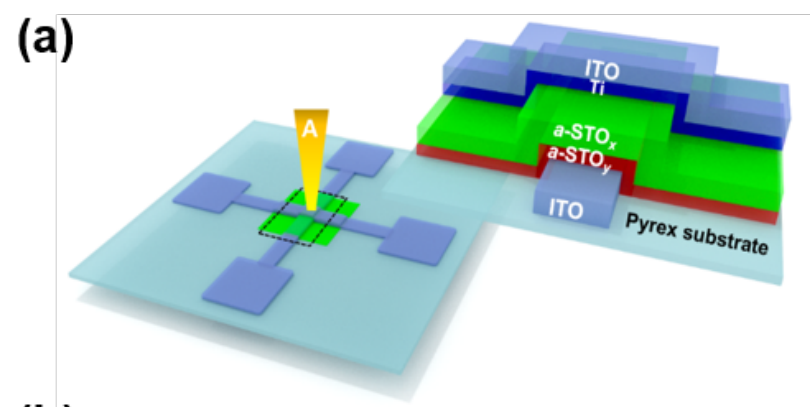

(b)

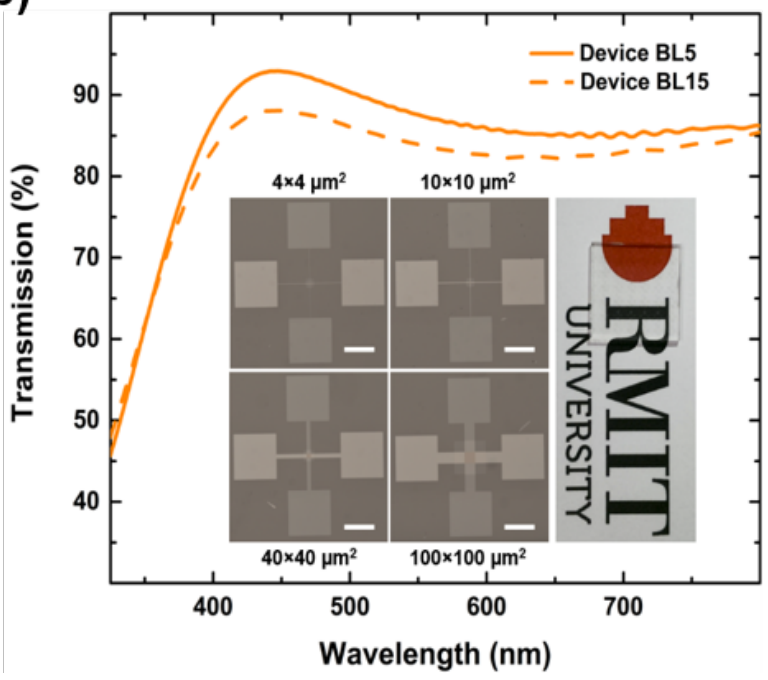

Fig 1. Physical structure and optical transmission characterization of $t$-ReRAM cells (a) Schematic illustrations of the fabricated cross-point devices. Area enclosed in rectangle (presented with black dashed outline) on the cross-point structure, represents the active $\mathrm{ITO} / \mathrm{Ti} / a-\mathrm{STO}_{x} / a-\mathrm{STO} / / \mathrm{ITO} /$ substrate region of the $t$-ReRAM cell. (b) The UV-Vis transmission spectra of Device BL15 and Device BL5 in the 325$800 \mathrm{~nm}$ optical range. The spectra are collected from the device area highlighted by " $A$ " in (a). Inset shows the optical microscope images of cross-points with difference sizes (scale bar $300 \mu \mathrm{m}$ ) and a $14 \times 14 \mathrm{~mm}^{2}$ sample placed on the RMIT University logo.
Two types of devices with varying $\mathrm{BL}\left(a-\mathrm{STO}_{y}\right)$ thicknesses $(15 \mathrm{~nm}$ and $5 \mathrm{~nm})$ are synthesized. We refer to these as Device BL15 (with the bilayer stack of $a-\mathrm{STO}_{x} / a-\mathrm{STO}_{y}$ $(10 \mathrm{~nm} / 15 \mathrm{~nm}))$ and Device BL5 (with the bilayer stack of $a$ $\left.\mathrm{STO}_{x} / a-\mathrm{STO}_{y}(10 \mathrm{~nm} / 5 \mathrm{~nm})\right)$. These devices are characterized optically and electrically. The optical transmission characteristics of the $t$-ReRAM cells are obtained from the area schematically indicated by a marker ' $A$ ' in Fig. 1a. To assess the optical properties of each component constituting a $t$-ReRAM cell, the transmission and reflectance characteristics of the sputtered oxides are also acquired (see Supporting Information, Fig. S5 and Fig. S6). The UV-Vis transmission spectra of the fabricated $t$-ReRAM cells (Fig. 1b) for both devices show $>80 \%$ transmission for visible optical wavelengths. Compared to Device BL5, a slightly lower transmission (by 3-5\%) is observed in Device BL15, which can be attributed to the difference in the thickness of the functional oxide stack.

\section{Compositional analyses of $a$-STO oxides}

To evaluate the chemical composition of the sputtered $a$-STO oxides, X-ray photoelectron spectroscopy (XPS) analysis is carried out on the uncapped bare $a-\mathrm{STO}_{x}$ and $a-\mathrm{STO}_{y}$ thin films. For elemental analysis, the as-obtained spectra are resolved by using a non-linear least square fitting method followed by background correction carried out using Shirley algorithm. ${ }^{17}$ The core level spectra of $\mathrm{O} 1 \mathrm{~s}$ and $\mathrm{Sr} 3 d$ do not show any significant shift in their respective binding energies (except for intensities) and are within the margin of error for the instrument resolution $( \pm 0.1 \mathrm{eV})$, as seen in the Supporting Information (Fig. S7). The analyses of the de-convoluted spectra of the core level binding energies of $\mathrm{Ti} 2 \mathrm{p}$ are shown in Fig. 2a. Both $a-\mathrm{STO}_{x}$ and $a-\mathrm{STO}_{y}$ spectra are fitted with two distinct components, namely $-\mathrm{Ti}^{4+}$ and $\mathrm{Ti}^{(4-\delta)+}$. It is known that the presence of solely a $\mathrm{Ti}^{4+}$ component corresponds to a fully stoichiometric STO oxide, while the $\mathrm{Ti}^{(4-\delta)+}$ species (such as $\mathrm{Ti}^{3+}$ and $\mathrm{Ti}^{2+}$ ) represent the presence of $\mathrm{V}_{\mathrm{o}} \mathrm{S}$ in the oxide system. However, $\mathrm{Ti}^{3+}$ and $\mathrm{Ti}^{2+}$ components cannot be resolved without ambiguity. ${ }^{18}$ Therefore to avoid any doubt in assigning the oxidation states to these reduced species, we fit with only one component at the lower binding energies and denote as $\mathrm{Ti}^{(4-\delta)+}$ (Fig. 2a). In our $a-\mathrm{STO}_{x}$ and $a-\mathrm{STO}_{y}$ oxides, the peaks at $\mathrm{Ti} 2 p_{3 / 2}$ binding energies of $458.4 \mathrm{eV}$ are assigned to $\mathrm{Ti}^{4+}$ oxidation state while the peaks at $456.2 \mathrm{eV}$ and $456.5 \mathrm{eV}$ are assigned to $\mathrm{Ti}^{(4-\delta)+}$ species. ${ }^{9}, \quad 19-21$ The relative concentration of the individual $\mathrm{Ti}^{4+}$ and $\mathrm{Ti}^{(4-\delta)+}$ species are calculated by integrating the fitted peaks. The relative concentrations of $\mathrm{Ti}^{4+}$ and $\mathrm{Ti}^{(4-\delta)+}$ in $a-\mathrm{STO}_{x}$ are calculated to be $72.9 \%$ and $27.1 \%$, respectively. On the other hand, the relative concentrations of $\mathrm{Ti}^{4+}$ and $\mathrm{Ti}^{(4-}$ ס)+ species in $a-\mathrm{STO}_{y}$ are calculated to be $75.2 \%$ and $24.8 \%$, respectively. It is seen that the concentration of $\mathrm{Ti}^{(4-\delta)+}$ species in $a-\mathrm{STO}_{x}$ is comparatively higher than in the $a-\mathrm{STO}_{y}$. This is expected, as the formation of a $\mathrm{Ti}^{(4-\delta)+}$ (i.e., an oxygen vacancy) due to the broken $\mathrm{Ti}-\mathrm{O}$ bond in the sub-lattice results in the donation of extra electrons into the Ti $3 d$ conduction band. ${ }^{22,23}$ 

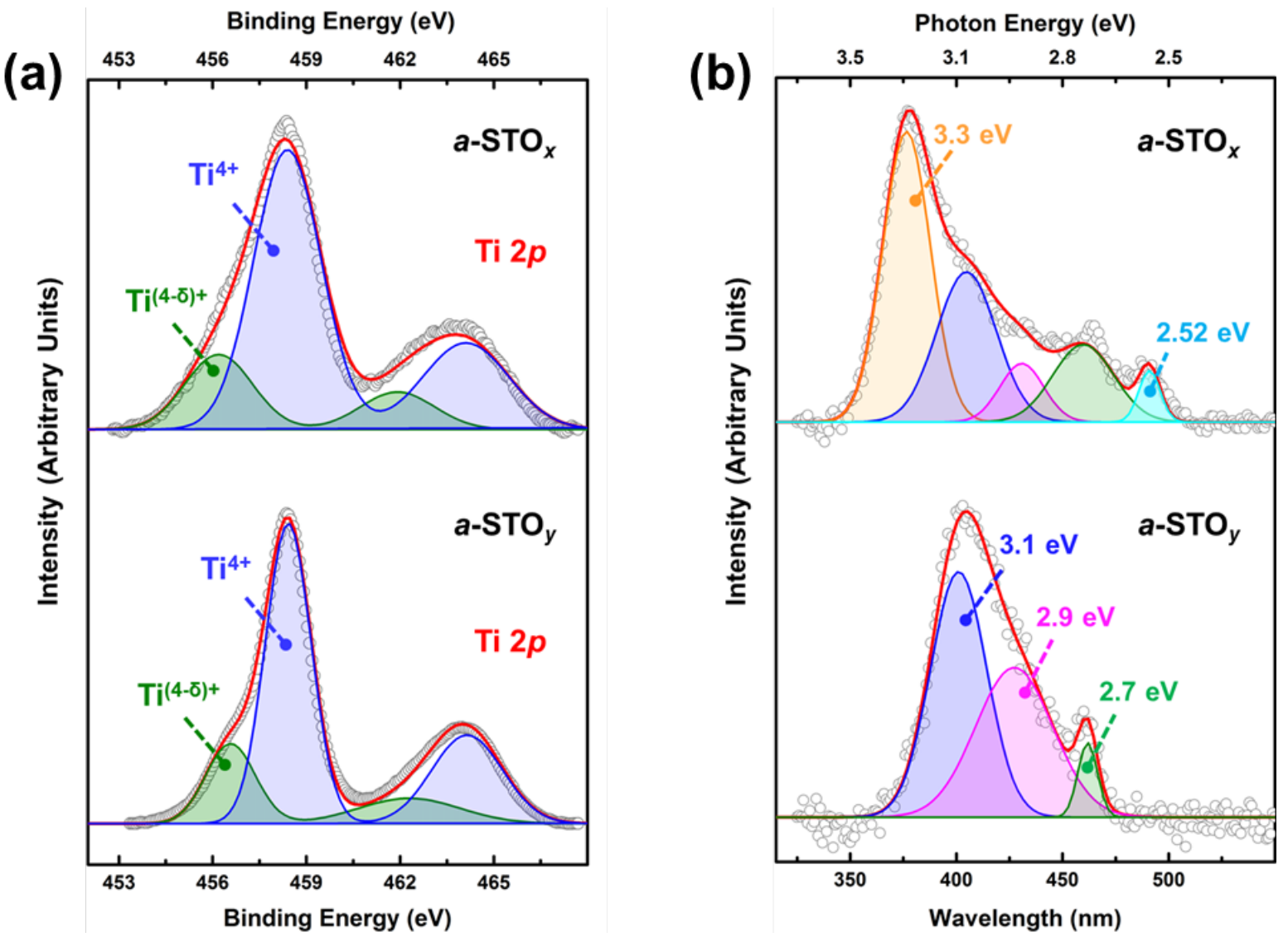

Fig. 2 Material characterization of the sputtered $a$-STO oxide thin films. (a) The resolved core-level X-ray photoelectron spectra of Ti $2 p$ of the sputtered $a$-STO thin films. (b) The resolved photoluminescence emission spectra of $a$-STO thin films, obtained at $300 \mathrm{~nm}$ excitation wavelength.

As such, the conduction process through the oxygen deficeint oxides is expected to take place through the $\mathrm{Ti}$ sub-oxide defect structures. ${ }^{24}$ This indicates that the sputtering of $a$-STO in an oxygen depleted environment results in a relatively higher concentration of $\mathrm{V}_{\mathrm{o}} \mathrm{s}$ than in the presence of oxygen. To explore the electronic structure of the deposited $a$-STO, photoluminescence $(\mathrm{PL})$ emission spectra are obtained at room temperature using excitation at $300 \mathrm{~nm}$ (Fig. 2b). Both oxides reveal a broad emission spectra which is characteristic of amorphous (doped and un-doped) STO. ${ }^{25-30}$ It is known that sputter deposition in a controlled oxygen environment leads to the reduction of the inherent oxygen vacancy related defect structures (i.e., $\mathrm{Ti}^{(4-\delta)+}$ species), ${ }^{9}$ which is confirmed with our observations in the XPS analysis (explained earlier). Therefore, $a-\mathrm{STO}_{x}$ is expected to show PL emission at lower wavelengths compared to $a-\mathrm{STO}_{y}$, which is indeed the case in our observations. De-convolution of the collected emission spectra from both $a$-STO films reveals multiple in-gap electronic states in $a$-STO oxides. ${ }^{28,} 31,32$ The $a$-STO ${ }_{x}$ thin film exhibits a broad emission spectrum between $340 \mathrm{~nm}$ and $500 \mathrm{~nm}$ with a maximum intensity at $376 \mathrm{~nm}(\sim 3.3 \mathrm{eV})$. On the other hand, the $a-\mathrm{STO}_{y}$ thin film shows emission peaks between $360 \mathrm{~nm}$ to
$480 \mathrm{~nm}$ with maximum intensity centered at $401 \mathrm{~nm}(\sim 3.1 \mathrm{eV})$. The observed red shift (of $25 \mathrm{~nm}$ ) in the main PL emission peak of $a-\mathrm{STO}_{y}$ (as compared to $a-\mathrm{STO}_{x}$ ) can be associated with the re-organization of defect levels in the $a-\mathrm{STO}_{y}$ oxide. ${ }^{24,26}$

\section{Resistive switching performance}

The typical bipolar resistive switching characteristics of Device BL15 (with $a-\mathrm{STO}_{x}(10 \mathrm{~nm}) / a-\mathrm{STO}_{y}(15 \mathrm{~nm})$ functional oxide stack) are shown in Fig. 3. In their virgin state, the memory cells show a highly insulating nature (virgin state resistance of at least $0.5 \mathrm{G} \Omega$ measured at $0.5 \mathrm{~V}$ ) and require an irreversible electroforming sweep to initialize the resistive switching behavior (see Supporting Informtion, Fig. S8). The current-voltage $(I-V)$ characteristics of Device BL15 show a reproducible cyclic bipolar resistive switching (Fig. 3a). Fig. 3b shows the statistical distribution of threshold voltages for the onset transition of SET (transition from HRS to LRS, $V_{\text {SET }}$ ) and RESET (transition from LRS to HRS, $V_{\text {RESET }}$ ) during the cyclic bipolar resistive switching (Fig. 3a), respectively. The distribution of $V_{S E T}$ ranges from -1 to $-2.7 \mathrm{~V}$ with a mean at $1.7 \mathrm{~V}$ while $V_{\text {RESET }}$ ranges from 1.6 to $4.9 \mathrm{~V}$ with a mean of $2.8 \mathrm{~V}$. 

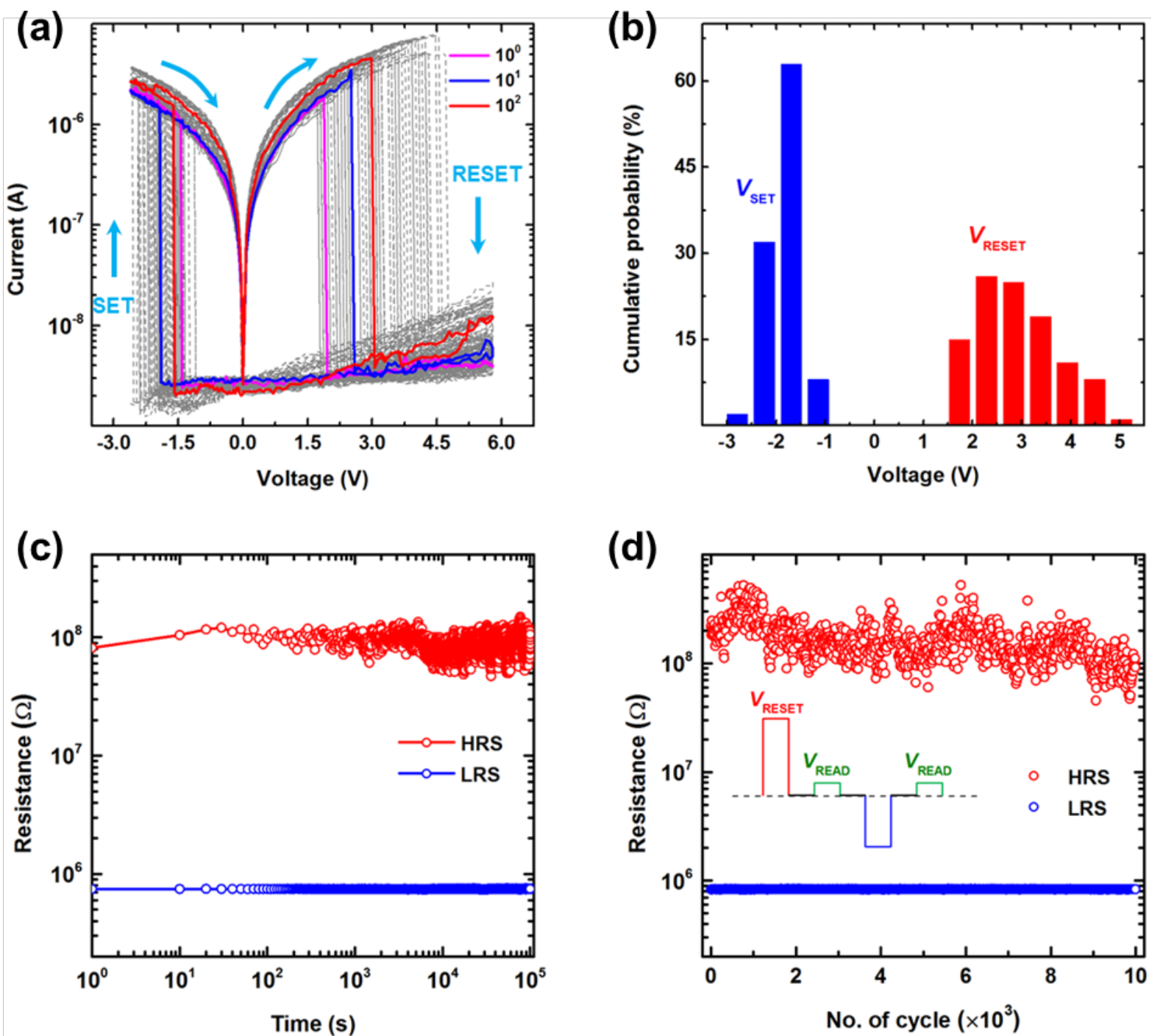

Fig 3. Electrical characterization of Device BL15 with $10 \times 10 \mu \mathrm{m}^{2}$ cell area. (a) The post-electroforming bipolar $I-V$ characteristics of 100 consecutive cycles on a semi-logarithmic scale. (b) Statistical distribution of $V_{\text {SET }}$ and $V_{\text {RESET }}$ for 100 consecutive switching cycles, shown in (a). (c) Retention of both LRS and HRS over $10^{5} \mathrm{~s}$ under a constant positive $V_{\text {READ }}$ of $0.5 \mathrm{~V}$. (d) Endurance over $10^{4}$ switching cycles. Inset shows the voltage pulse train used for $V_{\text {RESET }}(6.2 \mathrm{~V}), V_{\text {READ }}(0.5 \mathrm{~V})$ and $V_{\text {SET }}(-3.7 \mathrm{~V})$ during the endurance measurement where each pulse has a duration of $150 \mu \mathrm{s}$.

Although there are minor fluctuations in both LRS and HRS, the average switching ratio (HRS/LRS) is $\sim 119$ over a period of $10^{5} \mathrm{~s}$ under a constant positive $V_{\text {READ }}$ of $0.5 \mathrm{~V}$ (Fig. $3 \mathrm{c}$ ). The endurance of Device BL15 is measured for $10^{4}$ switching cycles by using pulsed WRITE/READ/ERASE cycles, as shown in Fig. 3d. During each switching cycle, short pulses (with duration of $150 \mu \mathrm{s})$ are used to RESET (6.2 V), READ (0.5 V) and SET $(-3.7 \mathrm{~V})$ the $t$-ReRAM cell.

The observed bipolar $I-V$ characteristics in Fig. 3a reveal that Device BL15 exhibits a linear LRS behavior under the measurement conditions. For large scale memory integration, such as crossbar ReRAM arrays, a linear LRS behavior makes the memory cells prone to the sneak paths and compromises the reliability of read data. ${ }^{33}$ However, this problem can be overcome by introducing non-linearity into the LRS. ${ }^{34}$ In our bilayer $t$-ReRAM cells it is expected that the $a$-STO $y$ layer plays a significant role in defining the resistive switching behavior. ${ }^{9}$ Therefore, to illustrate the role of the $a-\mathrm{STO}_{y}$ layer in our bilayer $t$-ReRAM cells, another device (designated as Device BL5) with three times less thickness of $a-\mathrm{STO}_{y}(5 \mathrm{~nm}$ in this case, instead of $15 \mathrm{~nm}$ ) as compared to Device BL15 is fabricated and characterized.

Fig. 4 shows the bipolar resistive switching characteristics of Device BL5. The $I-V$ characteristics (Fig. $4 a$ and 4 b) reveal an asymmetric bipolar resistive switching behavior. The peculiar $I-V$ crossing during the negative SET sweeps (at approximately $-2.5 \mathrm{~V}$ ) is explained in the later section. The non-linearity in onstate for Device BL15 and Device BL5 has been obtained by calculating the corresponding slopes of the $I-V$ curves between 0-3 $V$ (where the devices are in LRS). This slope for Device BL5 and Device BL15 is calculated to be $2 \mathrm{~V} /$ decade and 2.7 V/decade, respectively. This implies that Device BL5 

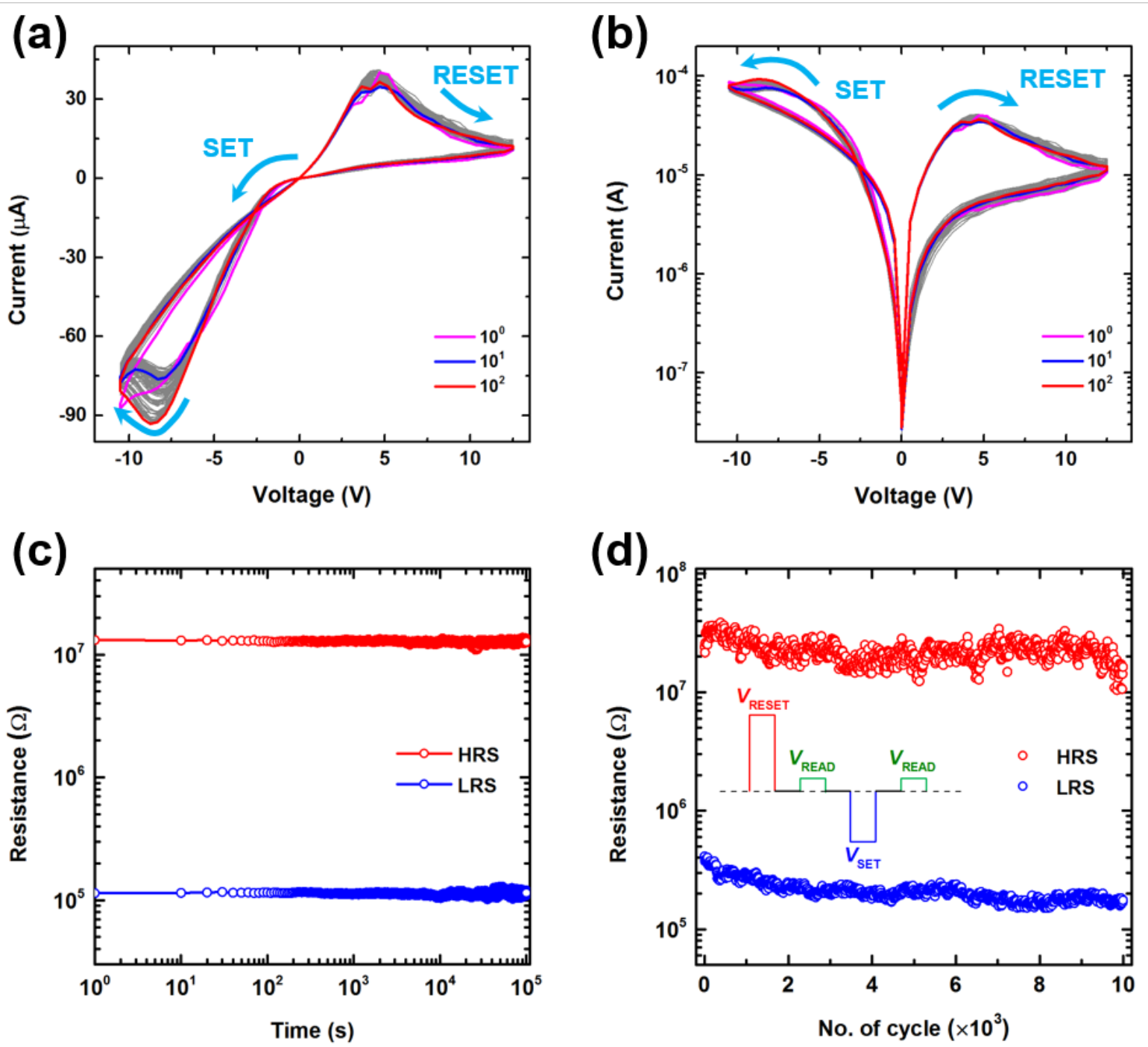

Fig 4. Electrical characterization of Device BL5 with $10 \times 10 \mu \mathrm{m}^{2}$ cell area. (a) The post-electroforming bipolar $I-V$ characteristics on a linear scale and (b) on a semi-log scale for 100 consecutive switching cycles. (c) Retention of both LRS and HRS over $10^{5} \mathrm{~s}$ under a $V_{\text {READ }}$ of $0.5 \mathrm{~V}$. (d) Endurance over $10^{4}$ switching cycles. Inset shows the voltage pulse train used for $V_{\text {RESET }}(13.8 \mathrm{~V}), V_{\text {READ }}(0.5 \mathrm{~V})$ and $V_{\text {SET }}(-12.7 \mathrm{~V})$ during the endurance measurement where each pulse has a duration of $500 \mu \mathrm{s}$.

exhibits relatively more non-linear characteristics than Device BL15.

The reliability characterization of Device BL5 reveals stable LRS and HRS retention (Fig. 4c) for $10^{5} \mathrm{~s}$ under a constant positive $V_{\text {READ }}$ of $0.5 \mathrm{~V}$. The average switching ratio is calculated to be $\sim 114$ over a period of $10^{5} \mathrm{~s}$. The endurance characteristics (Fig. 4d) measured for $10^{4}$ switching cycles show no device failure under a $V_{\text {READ }}$ of $0.5 \mathrm{~V}$. The non-linear LRS behavior observed in Device BL5 (Fig. 4a and 4b) is preferable to alleviate the sneak currents in their large scale ReRAM array integration. ${ }^{35}$ Generally, the memory cells responsible for sneak paths in an array experience lower voltage drop than $V_{\text {READ }}$ applied to a target cell. Thus, the non-linear dependence of read current on voltage can significantly reduce the contribution of sneak currents.

\section{Microstructure of $t$-ReRAMs}

To analyze the physical structure of the $t$-ReRAM cells, crosssectional TEM micrographs of virgin and electroformed memristive cells are obtained. Fig. 5 a shows the crosssectional TEM micrograph of Device BL5 in its virgin state. A high-resolution TEM (HRTEM) micrograph reveals an amorphous structure of the $a$-STO stack in the virgin $t$-ReRAM cell (inset of Fig. 5a). The selected area electron diffraction (SAED) pattern collected from the top ITO electrode and the $\mathrm{Ti} / a-\mathrm{STO}_{x}$ interface shows a diffused ring (Fig. 5b), indicative of an amorphous structure whereas, the SAED patterns of the post-deposition annealed bottom ITO electrode showed discrete diffraction spots (Fig. 5c). The diffraction patterns in Fig. $5 c$ can be indexed to the cubic bixbyite structure of $\ln _{2} \mathrm{O}_{3} .^{36-39}$ The polycrystalline structure of the post-deposition annealed ITO thin films is also identified by X-ray diffraction 

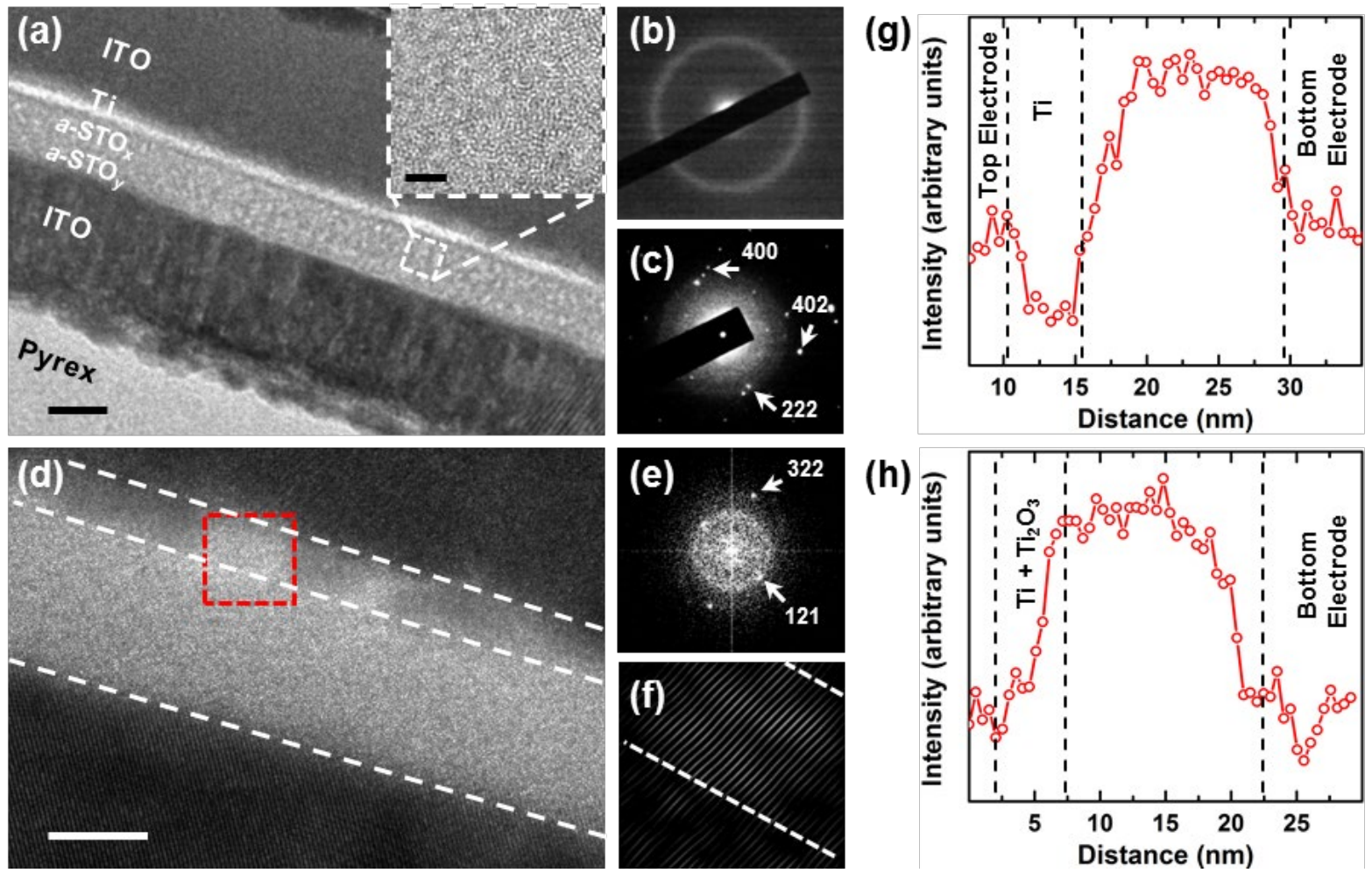

Fig. 5 Structural analysis of the $t$-ReRAM cells. (a) The cross-sectional TEM micrograph of a virgin Device BL5 $t$-ReRAM cell (scale bar $20 \mathrm{~nm}$ ). Inset shows the HRTEM image of $a$ $\mathrm{STO}_{x}$ layer (scale bar $2 \mathrm{~nm}$ ). The SAED patterns collected from the top as-deposited ITO electrode (b) and the bottom annealed ITO electrode (c). (d) The cross-sectional HRTEM micrograph of an electroformed Device BL5 $t$-ReRAM cell (scale bar $10 \mathrm{~nm}$ ). (e) The FFT of a region of interest selected and highlighted in (d). (f) The inverse FFT of the region of interest obtained from the diffraction spot corresponding to 121 in (e). (g) The EELS oxygen $K$-edge spectra of a virgin and (h) an electroformed $t$-ReRAM cell, recorded along a line scan across the cells.

(Supporting Information, Fig. S9). The cross-sectional HRTEM micrograph of an electroformed Device BL5 $t$-ReRAM cell (Fig. $5 \mathrm{~d}$ ) reveals the presence of a polycrystalline layer at the top $\mathrm{Ti} / a-\mathrm{STO}_{x}$ interface. For the compositional analyses of this polycrystalline layer, a region of interest (ROI) is selected (highlighted by a box in Fig. $5 \mathrm{~d}$ ) at the top $\mathrm{Ti} / a-\mathrm{STO}_{x}$ interface. Fig. 5e shows the Fast Fourier transform (FFT) diffraction pattern obtained from the ROI. The diffraction spots with the $d$-spacing of $1.8 \AA$ and $2.7 \AA$ are indexed to [322] and [121] planes of rhombohedral $\mathrm{Ti}_{2} \mathrm{O}_{3}$. The [121] diffraction spots are used to generate the inverse FFT (Fig. 5f) which highlights the dominant existence of $\mathrm{Ti}_{2} \mathrm{O}_{3}$ [121] in the $\mathrm{ROI}$. This indicates that during the electroforming process, the applied electric field induced Joule heating and diffusion of evolved oxygen ions into the $\mathrm{Ti}$ layer result in the formation of a polycrystalline $\mathrm{Ti}_{2} \mathrm{O}_{3}$ layer.

The observed brighter contrast at the top ITO/Ti/a-STO ${ }_{x}$ interface (in Fig. 5a) and the distribution of oxygen content across the virgin and electroformed $t$-ReRAM cells is assessed by the electron energy loss spectroscopy (EELS). The oxygen $K$ edge spectrum (Fig. $5 \mathrm{~g}$ ) collected along a line scan across the virgin $t$-ReRAM cell reveals the partial oxidation of $\mathrm{Ti}$ to a oxygen depleted titanium oxide layer (i.e., $\mathrm{Ti}_{2} \mathrm{O}_{3}$ ) at the top $\mathrm{Ti} / a-\mathrm{STO}_{x}$ interface. This observation is consistent with our earlier XPS analysis of virgin memory cells. ${ }^{9}$ On the other hand, the oxygen $K$-edge spectrum of the electroformed $t$-ReRAM cell (Fig. 5h) shows an increase in the thickness of polycrystalline $\mathrm{Ti}_{2} \mathrm{O}_{3}$ layer at the top $\mathrm{Ti} / a-\mathrm{STO}_{x}$ interface and a decrease in the oxygen content at the bottom $a-\mathrm{STO}_{y} / \mathrm{ITO}$ interface as compared to rest of the $a$-STO stack. It can be inferred that relatively higher concentration of $\mathrm{V}_{\mathrm{o}} \mathrm{s}$ are present at the vicinity of bottom interface due to the reversible redox reactions (under the influence of electroforming bias). Furthermore, the $\mathrm{Ti} L_{2,3}$-edge spectrum collected from the electroformed cell showed relatively higher morphological ordered in $a$-STO than the virgin $t$-ReRAM cell (Supporting Information, Fig. S10). A detailed description of the electronic structure of the $a$-STO layers and switching mechanism of the $t$-ReRAM cells is explained in rest of this work.

\section{Resistive switching mechanism in $t$-ReRAMs}

In order to assess the resistive switching mechanism in our bilayer $t$-ReRAM cells, a room-temperature $\mathrm{PL}$ mapping technique is employed due to its non-destructive analysis nature (unlike cross-sectional TEM analyses) and sensitivity to the defect or impurity states. ${ }^{25}$ It is well-known that the intrinsic oxygen related defects (such as $\left[\mathrm{TiO}_{5} \cdot \mathrm{V}_{\mathrm{o}}^{1+}\right]$ and $\left[\mathrm{TiO}_{5} \cdot \mathrm{V}_{\mathrm{o}}^{2+}\right]$ complexes, where $\mathrm{V}_{\mathrm{o}}^{1+}$ and $\mathrm{V}_{\mathrm{o}}^{2+}$ denote single and double positively charged oxygen vacancies, respectively) in $a$ STO introduce localized in-gap states above the valance band and are responsible for the room temperature PL emission. ${ }^{23,}$ 26, 30, 32 Also, the $\mathrm{PL}$ emission is correlated with the 

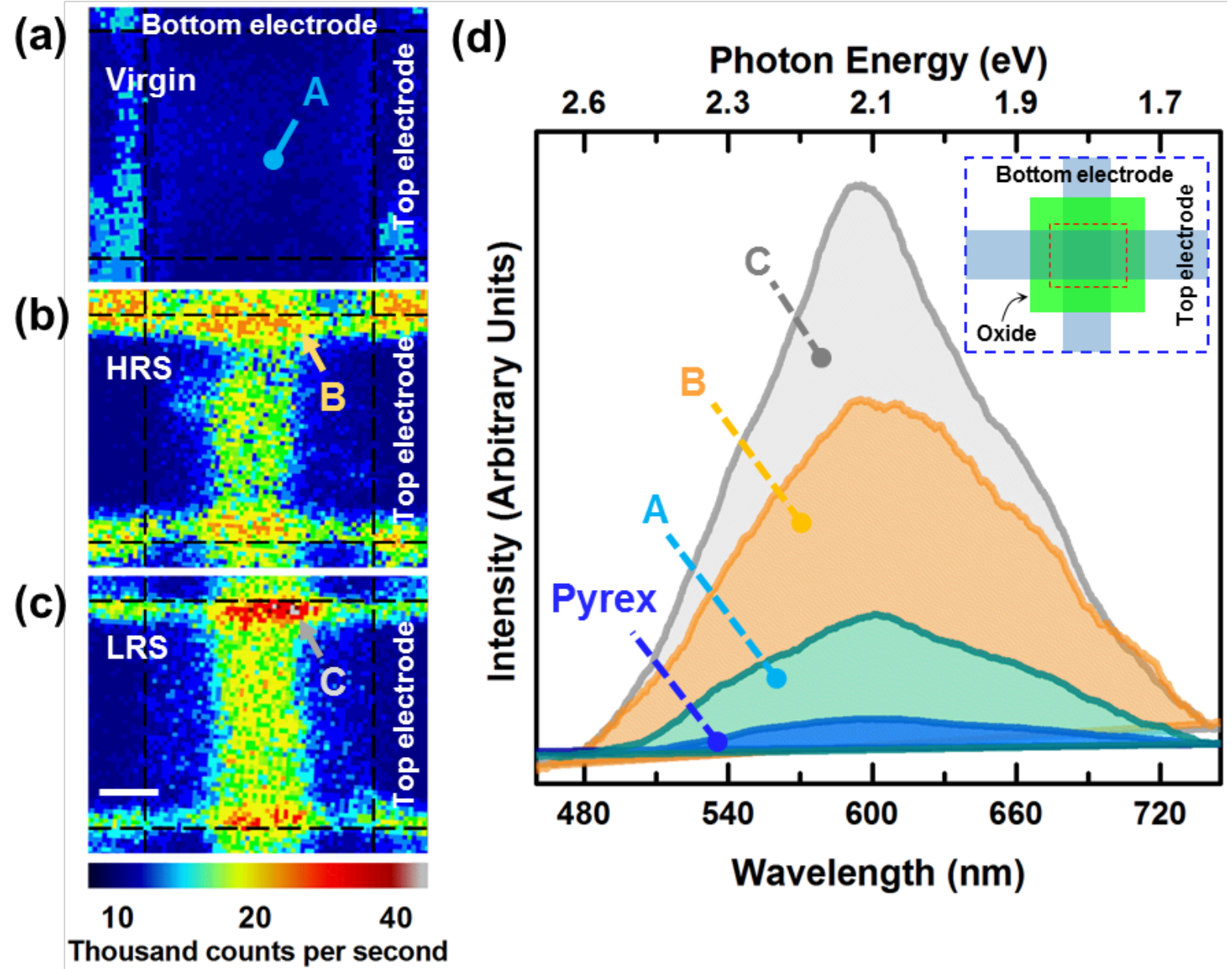

Fig. 6 Photoluminescence mapping of the bilayer $t$-ReRAM cell. The PL maps (top-view) of a $4 \times 4 \mu m^{2}$ Device BL15 $t$-ReRAM in its (a) virgin state, (b) HRS and (c) LRS, under $405 \mathrm{~nm}$ excitation source. Scale bar corresponds to $1 \mu \mathrm{m}$. (d) The PL emission spectra collected from different regions of the cell in its virgin, HRS and LRS marked in (a), (b) and (c), respectively. Inset of (d) shows a schematic of a $t$-ReRAM cell highlighting the region of interest used for PL maps in (a)-(c).

concentration of oxygen related defects (i.e., $\mathrm{V}_{\mathrm{o}} \mathrm{s}$ ) such that the $\mathrm{PL}$ response can be modulated by changing the density of $\mathrm{V}_{\mathrm{o}} \mathrm{s}^{25,31,40}$ By exploiting the high sensitivity of PL emission to the $V_{o} s$, we demonstrate direct observation of oxygen deficient regions and conductive filament in our bilayer $t$ ReRAM cells (Fig. 6) via submicron high spatial resolution PL maps, under a $405 \mathrm{~nm}$ excitation source (see Experimental). As observed in XPS and PL analyses of the sputtered $a$-STO thin films (Fig. 2) both as-grown oxides $\left(a-\mathrm{STO}_{x}\right.$ and $a$-STO $\left.{ }_{y}\right)$ are oxygen deficient. So, the identification of oxygen deficient regions by $\mathrm{PL}$ signals is expected in a virgin $t$-ReRAM cell. However, diffuse scattering in ITO/glass substrate hinders the identification of oxygen deficient regions in the virgin cell (Fig. 6a). On the other hand, a switching cell shows the distribution of oxygen deficient regions over the cell area in both HRS and LRS with different PL emission intensities (Fig. 6b and $c$, respectively). This indicates that the overall concentration of $\mathrm{V}_{\mathrm{o}} \mathrm{s}$ increased in the bilayer stack after the electroforming and subsequent switching cycles. The intensity thresholds are kept the same to enable a direct comparison of the PL response in different resistive states. On comparing the PL maps, relatively more oxygen deficient regions are observed at the edges of the top electrode after a virgin cell is subjected to the electroforming process. This can be attributed to the applied electric field induced generation of higher concentration of $\mathrm{V}_{o} \mathrm{~S}$ in these regions during the electroforming. ${ }^{41,} 42$ Fig. $6 \mathrm{~d}$ shows broad peaks of the $\mathrm{PL}$ emission spectra around $600 \mathrm{~nm}(2.1 \mathrm{eV})$, collected from different regions of the $t$-ReRAM cell in its virgin, HRS and LRS marked as ' $A$ ', ' $B$ ', and ' $C$ ' in Fig. $6 a, b$ and $C$, respectively. Comparatively, the highest PL intensity observed for location ' $C$ ' (Fig. 6c) can be ascribed to the accumulation of $V_{o} S$ at this hotspot under the influence of the applied LRS bias. This localized intense PL emission compared to its vicinity indicates the presence of a conducting filament comprising of accumulated $V_{o S}$ in the LRS. While relatively lower PL emission (' $B$ ') from the same region, indicates a change in the distribution of $V_{o} S$ in HRS. Recently, by using different spectroscopic and nano-contact studies, we have shown the existence of nano-filaments in $a$-STO based resistive memories. ${ }^{9,} 16,43$ However, this PL mapping technique further highlights the primary role of $\mathrm{V}_{\mathrm{o}} \mathrm{s}$ in disordered $a$-STO oxides and clearly indicates the filamentary based resistive switching in our $a$-STO bilayer $t$-ReRAMs. 

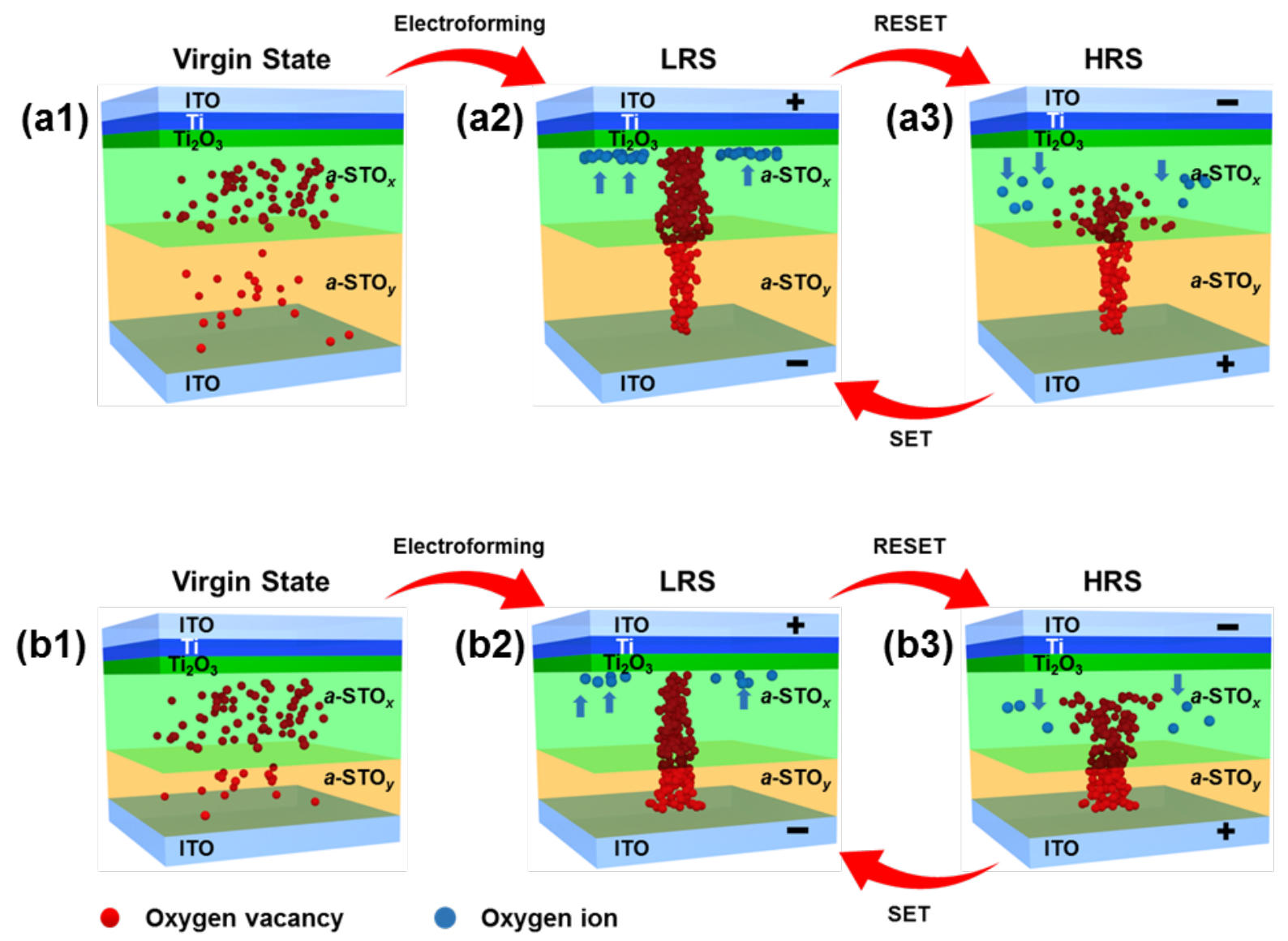

Fig. 7 Schematic illustrations of the possible resistive switching mechanisms in the $t$-ReRAM cells of (a1-a3) Device BL15 and (b1-b3) Device BL5. Transformation of the $t$-ReRAM cell from $(a 1, b 1)$ a virgin state to a repeatable $(a 2, b 2)$ LRS and $(a 3, b 3)$ HRS. The red and blue spheres represent oxygen vacancies $\left(V_{0} s\right)$ and oxygen ions $\left(O^{2-}\right)$, respectively, whereas the blue arrows show the direction of $\mathrm{O}^{2-}$ drift under the influence of applied electric field.

Based on the electrical and material characterization of our bilayer homojunction $t$-ReRAM cells, we propose a possible resistive switching mechanism in these devices. It is well known that mixed electronic/ionic charge transport and reversible redox reactions are mainly responsible for the resistive switching in STO based ReRAMs. ${ }^{9}, 11,16,44$ In the presence of an externally applied electric field, reversible redox reactions increase the concentration of as-grown $V_{o}$ based defect structure in the $\mathrm{Ti}-\mathrm{O}$ sub-lattice and consequently nanoionic charge transportation occurs along the $\mathrm{Ti}^{3+}$ suboxide. ${ }^{24,42}$ As evident from the XPS analysis, the relative concentration of as-grown $\mathrm{Ti}^{(4-\delta)+}$ species is higher in $a-\mathrm{STO}_{x}(27.1 \%)$ than $a-\mathrm{STO}_{y}(24.8 \%)$; therefore, the charge carriers (e.g., oxygen ions and free electrons) are expected to drift across the $a$-STO bilayer stack under the influence of an applied bias. So the proposed resistive switching model in our bilayer $t$-ReRAM cells is based on the extension and rupture of $V_{0}$ based defect structure (i.e., localized conductive filament) through the $a$-STO layers. ${ }^{44,45}$

Fig. 7 schematically illustrated the possible resistive switching mechanism in our bilayer homojunction (Device BL15 and Device BL5) $t$-ReRAM cells. In its virgin state, a $t$-ReRAM cell (regardless of the device type) has higher concentration of asgrown $\mathrm{V}_{\mathrm{o}} \mathrm{S}$ in $a-\mathrm{STO}_{x}$ than $a-\mathrm{STO}_{y}$ layer (Fig. 7a1 and b1). As observed from the TEM analysis (Fig. 5) of a virgin $t$-ReRAM cell and our previous study, ${ }^{9}$ the top Ti layer partially oxidizes in the vicinity of $a-\mathrm{STO}_{x}$ and a thin layer of amorphous Ti suboxide (such as $\mathrm{Ti}_{2} \mathrm{O}_{3}$ ) is formed at $\mathrm{Ti} / a-\mathrm{STO}_{x}$ interface. However, this interfacial Ti sub-oxide layer is oxygen depleted and therefore the carrier transport can still take place across the top interface. ${ }^{9,} 19$ When the bottom ITO electrode of a virgin $t$-ReRAM cell is negatively biased (Fig. 7a2 and b2), the localized $\mathrm{Ti}-\mathrm{O}$ sites become oxygen depleted due to the electrochemical redox process and the oxygen ions $\left(\mathrm{O}^{2-}\right) \mathrm{drift}$ towards the top ITO electrode (acting as anode) through the $a-\mathrm{STO}_{x}$ layer. At the anode, $\mathrm{O}^{2-}$ discharge to evolve oxygen gas due to the oxidation reaction. ${ }^{41,42}$ In the presence of neutral oxygen and applied electric field induced Joule heating, the thickness of $\mathrm{Ti}_{2} \mathrm{O}_{3}$ layer increases and its microstructure changes to polycrystalline (as observed in cross-sectional TEM analyses, Fig 5d-f). Simultaneously, with an increasing negative bias at the bottom electrode, the concentration of $V_{o} S$ increases in the bilayer oxide stack (as observed in PL maps Fig 6). When the applied bias reaches the level of forming voltage for a virgin (or $V_{S E T}$ for an electroformed) $t$-ReRAM cell, the concentration of $\mathrm{V}_{\mathrm{o}} \mathrm{S}$ extends toward the anode. ${ }^{26,27} \mathrm{As}$ a result the bilayer $a-\mathrm{STO}_{x} / a-\mathrm{STO}_{y}$ stack provides a localized conductive path for the electronic transport between opposite 

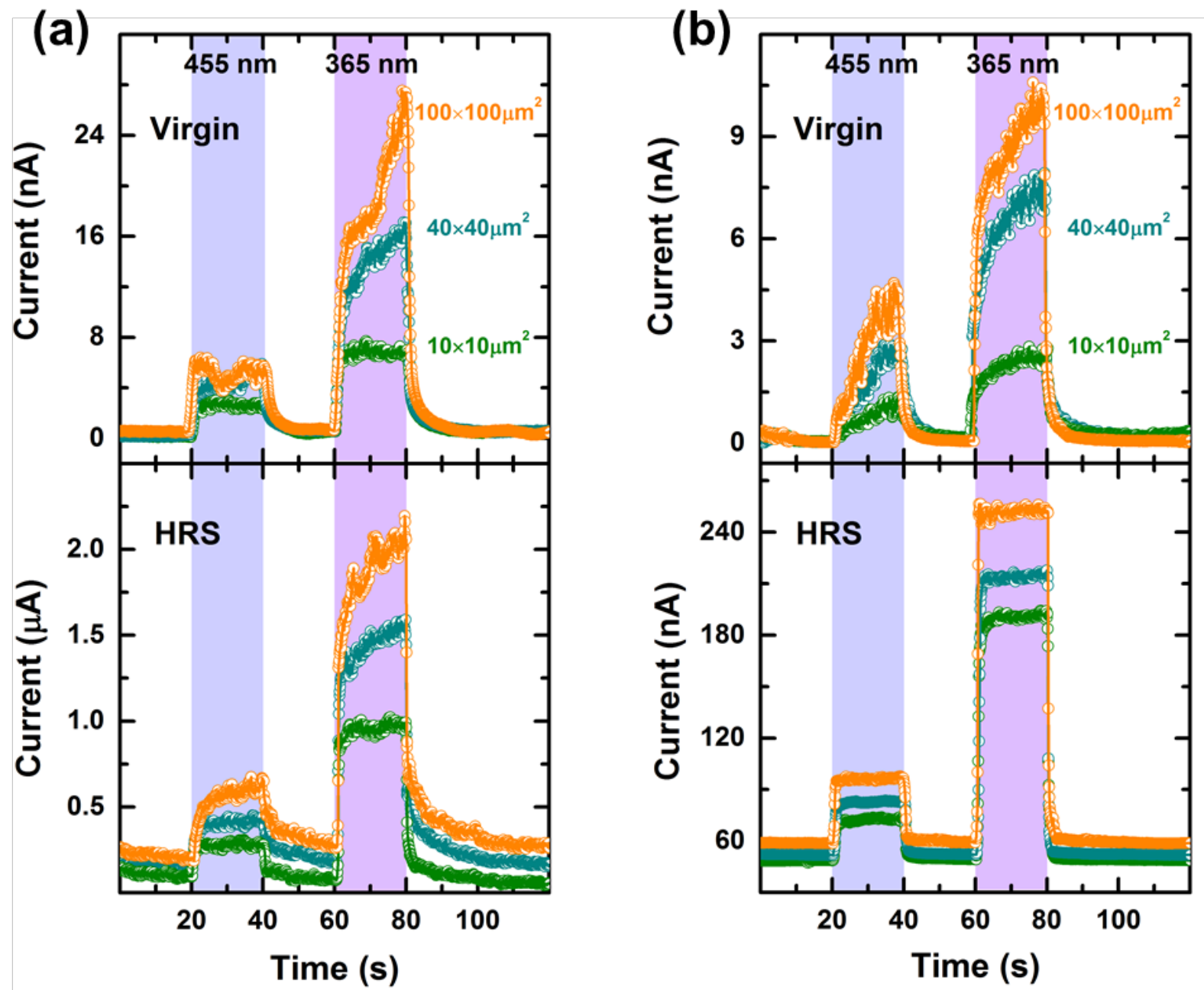

Fig 8. Photo-response of ITO/Ti/a-STO $x / a-\mathrm{STO}_{y} / \mathrm{ITO}$ structured $t$-ReRAM cells in virgin and HRS. (a) The measured photocurrent of Device BL15 and (b) Device BL5 upon exposure to the blue and UV illuminations with wavelengths of 455 and $365 \mathrm{~nm}$, respectively, at a $V_{\text {READ }}$ of $0.5 \mathrm{~V}$. The $t$-ReRAM cells with active area of $10 \times 10,40 \times 40$ and $100 \times 100 \mu \mathrm{m}^{2}$ are sequentially exposed to the illuminations with an incident power of $2.5 \pm 0.2 \mathrm{~mW} / \mathrm{cm}^{2}$ for $20 \mathrm{~s}$.

electrodes thereby, significantly changing virgin state resistance (or HRS for an electroformed $t$-ReRAM cell) to LRS. Also the Joule heating effect can increase the microstructural order of the amorphous oxides, ${ }^{46,47}$ as observed in our EELS analysis of the electroformed cell (Supporting Information, Fig. S10).

On the other hand, a negative bias on the top ITO electrode (acting as a cathode, Fig. $7 \mathrm{a} 3$ and b3) drifts the $\mathrm{O}^{2-}$ from the top $\mathrm{Ti}_{2} \mathrm{O}_{3} / a-\mathrm{STO}_{x}$ interface towards the $a-\mathrm{STO}_{x}$ layer. As the bias increases to $V_{\text {RESET, }}$ the $\mathrm{O}^{2-}$ recombine with the $V_{o} S$ and produces neutral oxygen ions $\left(\mathrm{O}^{2-}+\mathrm{V}_{\mathrm{o}} \rightarrow \mathrm{O}_{0}\right)$. Consequently, a partially ruptured conductive path remains in the bilayer $a-\mathrm{STO}_{x} / a-\mathrm{STO}_{y}$ stack and the $t$-ReRAM cell switches to HRS.

Despite the similar top and bottom interfaces $\left(\mathrm{Ti}_{2} \mathrm{O}_{3} / a-\mathrm{STO}_{x}\right.$ and $a-\mathrm{STO}_{y} / \mathrm{ITO}$, respectively), the observed difference in the resistive switching behaviors in Device BL15 (linear switching) and Device BL5 (non-linear switching) can be explained by considering the distribution of $\mathrm{V}_{0} \mathrm{~S}$ in the $a-\mathrm{STO}_{x}$ and $a-\mathrm{STO}_{y}$ layers. The Device BL15 electroforms at relatively higher voltages than Device BL5 (Supporting Information, Fig. S8) which results in higher concentration of $\mathrm{V}_{\mathrm{o}} \mathrm{S}$ in the $a-\mathrm{STO}_{x}$ layer, as depicted in Fig. 7a2. Consequently, the $a$-STO $\mathrm{ST}_{x}$ layer is more conductive than $a-\mathrm{STO}_{y}$ layer which translates into higher resistances in both LRS and HRS of Device BL15 (even at comparatively lower SET/RESET voltages than Device BL5). On the other hand, comparatively higher SET voltages of Device BL5 result in a drift of $\mathrm{V}_{\mathrm{o}} \mathrm{S}$ from the $a-\mathrm{STO}_{x}$ layer to the vicinity of bottom $a$-STO $/$ /TO interface (as depicted in Fig. 7b2). This explains the relatively higher conductivity of the $a-\mathrm{STO}_{y}$ layer of Device BL5 (than $a-\mathrm{STO}_{y}$ layer of Device BL15) and also its lower LRS/HRS resistances.

Furthermore, a plausible explanation for the peculiar $I-V$ crossing during the negative SET sweeps of Device BL5 (Fig. 4a and $b$ ), can be presented by considering the Joule heating induced electrochemical modification of the top $\mathrm{Ti}_{2} \mathrm{O}_{3} / a-\mathrm{STO}_{x}$ 
interface. Under a relatively high SET bias, the localised temperatue around the filamentary region can be significantly higher than the rest of device (due to the Joule heating). This can induce a repeatable electro-thermal negative differential resistance (NDR) effect which explains the decrease in current at $-8.2 \pm 0.5 \mathrm{~V}$ (Fig. $4 \mathrm{a}$ ). Since the $\mathrm{Ti}$ sub-oxide phase (such as $\left.\mathrm{Ti}_{4} \mathrm{O}_{7}\right)$ is known to exhibit the Joule heating induced NDR phenomenon, ${ }^{34,48}$ the top $\mathrm{Ti} / \mathrm{Ti}_{2} \mathrm{O}_{3} / a-\mathrm{STO}_{x}$ interface is likely to be electrochemically modified (to a sub-stoichiometeric interfacial layer) during the cyclic SET sweeps. This possibly underpins the peculiar $I-V$ crossing at approximately $-2.5 \mathrm{~V}$ in Device BL5, however a detailed investigation to experimentally prove its origin is a subject for future studies.

\section{Photo-response of $t$-ReRAMs}

To fully benefit from the transparency of the $t$-ReRAM cells, it is essential to explore the optical tunability of their resistive states. We studied the photo-response of our $t$-ReRAM cells via a sequential exposure to different illumination wavelengths. As observed in the PL spectra (Fig. 2b), the presence of $\mathrm{V}_{\mathrm{o}} \mathrm{s}$ induced in-gap electronic states result in a photo-response of $a$-STO oxides in a wide wavelength window (ranging between $365 \mathrm{~nm}$ to $530 \mathrm{~nm}$ ). This suggests that the $t$ ReRAM cells will be optically responsive to the excitation wavelengths in this range.

Fig. 8 shows the time-resolved photo-electronic response of our $t$-ReRAM cells with varying active areas to different wavelengths in their virgin and HRS states. It can be observed that regardless of their active cell area and resistive states, both Device BL15 (Fig. 8a) and Device BL5 (Fig. 8b) show higher photocurrent under ultraviolet (UV, $365 \mathrm{~nm}$ ) as compared to the blue $(455 \mathrm{~nm})$ under equal power densities of $2.5 \pm 0.2 \mathrm{~mW} / \mathrm{cm}^{2}$. This is consistent with the observed $\mathrm{PL}$ spectra from $a$-STO oxides and indicates that more photoinduced carriers are generated as the illumination wavelength decreases towards the corresponding bandgap $(<390 \mathrm{~nm}) .49-51$ Both Device BL15 and Device BL5 show an area dependent photocurrent in their virgin state. This can be explained by considering the area dependent density of $V_{o} s$ in virgin $t$ ReRAM cells and also relatively lower density of $V_{o} s$ (than electroformed cells) where they act as trapping sites for injected electrons under a low $V_{\text {READ. }}{ }^{52-54}$ Light illumination detrapps the electrons into the conduction band which are driven through the device by $V_{\mathrm{READ}}{ }^{2,}{ }^{55-57}$ On the other hand, no obvious relation between the active cell size and photocurrent is found in HRS of both Device BL15 and Device BL5 $t$-ReRAM cells. In HRS, low electrical conduction is usually attributed to the transportation of electronic carriers through the oxide/electrode interfacial barrier. ${ }^{10,41}$ While the area independent HRS photo-response in the $t$-ReRAM cells can be associated with the transportation of photo-excited electrons through the localized variation of ITO/a-STO interfacial barrier at the conductive filament without affecting the remaining interfacial barrier, ${ }^{58}$ it should be noted that photo-electronic response is only observed in HRS of the $t$ ReRAM cells. In the LRS however, the presence of electrically induced conductive filamentary path (as discussed in the preceding section) provides a fast route for the transportation of charge carriers; ${ }^{42}$ hence, diminishing the photo-response of the $t$-ReRAM cells. Such an area independent optical and electrical properties of $t$-ReRAMs highlight the scalability potential of these devices.

Regardless of the cell area, relatively higher average UV response (/uv/ / Dark $)$ of Device BL15 $(8.7 \pm 0.1)$ than Device BL5 $(4.1 \pm 0.2)$, can be attributed to the higher $V_{0}$ concentration owing to the thickness of $a$-STO stack. ${ }^{54,59,60}$ Furthermore, a fast photocurrent modulation $(10 \mathrm{~Hz})$ of Device BL15 is achieved under the UV illumination (Supporting Information, Fig. S11), indicating a fast response time, a required feature for photo-electronic devices such as optoelectronic switches and memories. ${ }^{59,} 61$ In addition, to assess the contribution of ITO electrodes in the photo-response, each electrode is exposed to the illumination and no significant photo-response is observed (Supporting Information, Fig. S12). As a result, the role of ITO can be neglected. These results reveal that the $a$-STO $t$ ReRAMs, with attractive features such as fast and area independent photo-response, demonstrate a multifunctional storage system where memory states can be optically read by using a specific light irradiation. Moreover, the reported memory design is also capable of enabling memory cells to work as light sensors.

\section{Conclusion}

In summary, we have demonstrated highly transparent ReRAM cells with bipolar switching behavior. A bilayer stack of amorphous STO is utilized as the memory element sandwiched between transparent ITO electrodes. Comprehensive optical, electrical and compositional characterizations are conducted to propose a switching mechanism for the devices. The thickness of the bilayer stack is also varied to study its influence on the switching performance and to control the linearity in the switching behavior. Devices can be designed to exhibit a non-linear LRS which highlights their potential for large scale integration of $t$-ReRAM array. Additionally, the memory devices are shown to exhibit a transient photoresponse in their HRS using a range of illumination wavelengths. Combining memristive properties with optically modulated HRS indicates the versatile functionality of such $t$-ReRAMs for optoelectronic applications such as photo detectors/sensors. As such, this study is a step towards the integration of transparent memory units into functional transparent electronics.

\section{Acknowledgements}

The authors acknowledge the Australian Research Council for funding in the form of project (DP130100062 and DP140103448), fellowship (DE160100023, FT140101285), and infrastructure (LE0882246 and LE0989615) support. The authors also acknowledge support from RMIT University's Enabling Capability Platforms initiative and technical assistance 
of the Micro Nano Research Facility and RMIT Microscopy and Microanalysis Facility.

\section{References}

1. J. Shang, G. Liu, H. Yang, X. Zhu, X. Chen, H. Tan, B. Hu, L. Pan, W. Xue and R.-W. Li, Adv. Funct. Mater., 2014, 24, 2171-2179.

2. H. Tan, G. Liu, X. Zhu, H. Yang, B. Chen, X. Chen, J. Shang, W. D. Lu, Y. Wu and R. W. Li, Adv. Mater., 2015, 27, 2797-2803.

3. M. Ungureanu, R. Zazpe, F. Golmar, P. Stoliar, R. Llopis, F. Casanova and L. E. Hueso, Adv. Mater., 2012, 24, 2496-2500.

4. A. Bera, H. Peng, J. Lourembam, Y. Shen, X. W. Sun and T. Wu, Adv. Funct. Mater., 2013, 23, 4977-4984.

5. J. Yao, Z. Jin, L. Zhong, D. Natelson and J. M. Tour, ACS Nano, 2009, 3, 4122-4126.

6. J. Yao, J. Lin, Y. Dai, G. Ruan, Z. Yan, L. Li, L. Zhong, D. Natelson and J. M. Tour, Nat. Commun., 2012, 3, 1101.

7. C. He, J. Li, X. Wu, P. Chen, J. Zhao, K. Yin, M. Cheng W. Yang, G. Xie, D. Wang, D. Liu, R. Yang, D. Shi, Z. Li, L. Sun and G. Zhang, Adv. Mater., 2013, 25, 55935598.

8. K. Roy, M. Padmanabhan, S. Goswami, T. P. Sai, G. Ramalingam, S. Raghavan and A. Ghosh, Nat. Nanotechnol., 2013, 8, 826-830.

9. H. Nili, S. Walia, A. E. Kandjani, R. Ramanathan, P. Gutruf, T. Ahmed, S. Balendhran, V. Bansal, D. B. Strukov, O. Kavehei, M. Bhaskaran and S. Sriram, Adv. Funct. Mater., 2015, 25, 3172-3182.

10. R. Dittmann, R. Muenstermann, I. Krug, D. Park, T. Menke, J. Mayer, A. Besmehn, F. Kronast, C. M. Schneider and R. Waser, Proc. IEEE, 2012, 100, 19791990.

11. R. Muenstermann, T. Menke, R. Dittmann and R. Waser, Adv. Mater., 2010, 22, 4819-4822.

12. Y. Yang, S. Choi and W. Lu, Nano Lett., 2013, 13, 29082915

13. M. J. Lee, C. B. Lee, D. Lee, S. R. Lee, M. Chang, J. H. Hur, Y. B. Kim, C. J. Kim, D. H. Seo, S. Seo, U. I. Chung, I. K. Yoo and K. Kim, Nat. Mater., 2011, 10, 625-630.

14. T. You, Y. Shuai, W. Luo, N. Du, D. Bürger, I. Skorupa, R. Hübner, S. Henker, C. Mayr, R. Schüffny, T. Mikolajick, O. G. Schmidt and H. Schmidt, Adv. Funct. Mater., 2014, 24, 3357-3365.

15. A. Siemon, T. Breuer, N. Aslam, S. Ferch, W. Kim, J. van den Hurk, V. Rana, S. Hoffmann-Eifert, R. Waser, S. Menzel and E. Linn, Adv. Funct. Mater., 2015, 25, 6414-6423.

16. H. Nili, S. Walia, S. Balendhran, D. B. Strukov, M. Bhaskaran and S. Sriram, Adv. Funct. Mater., 2014, 24, 6741-6750.

17. D. A. Shirley, Phys. Rev. B, 1972, 5, 4709-4714.

18. K. Zakrzewska, Adv. Mater. Sci. Eng., 2012, 2012, 13.

19. S. Stille, C. Lenser, R. Dittmann, A. Koehl, I. Krug, R. Muenstermann, J. Perlich, C. M. Schneider, U. Klemradt and R. Waser, Appl. Phys. Lett., 2012, 100, 223503.
20.
H. L. Cai, X. S. Wu and J. Gao, Chem. Phys. Lett., 2009, 467, 313-317.

P. A. W. v. d. Heide, Q. D. Jiang, Y. S. Kim and J. W. Rabalais, Surf Sci., 2001, 473, 59-70.

M. Scigaj, J. Gázquez, M. Varela, J. Fontcuberta, G. Herranz and F. Sánchez, Solid State lonics, 2015, 281, 68-72.

C. D. Pinheiro, E. Longo, E. R. Leite, F. M. Pontes, R. Magnani, J. A. Varela, P. S. Pizanni, T. M. Boschi and F. Lanciotti, Appl. Phys. A: Mater. Sci. Process., 2003, 77, 81-85.

K. Morii, H. Kawano, I. Fujii, T. Matsui and Y. Nakayama, J. Appl. Phys., 1995, 78, 1914.

D. Kumar and R. C. Budhani, Phys. Rev. B, 2015, 92.

E. Goldenberg, T. Bayrak, C. Ozgit-Akgun, A. Haider, S. A. Leghari, M. Kumar and N. Biyikli, Thin Solid Films, 2015, 590, 193-199.

H. Yasuda and Y. Kanemitsu, Phys. Rev. B, 2008, 77, 193202.

F. M. Pontes, E. Longo, E. R. Leite, E. J. H. Lee, J. A. Varela, P. S. Pizani, C. E. M. Campos, F. Lanciotti, V. Mastellaro and C. D. Pinheiro, Mater. Chem. Phys., 2003, 77, 598-602.

D. Kan, R. Kanda, Y. Kanemitsu, Y. Shimakawa, M Takano, T. Terashima and A. Ishizumi, Appl. Phys. Lett., 2006, 88, 191916.

L. E. B. Soledade, E. Longo, E. R. Leite, F. M. Pontes, F. Lanciotti Jr, C. E. M. Campos, P. S. Pizani and J. A. Varela, Appl. Phys. A: Mater. Sci. Process., 2002, 75, 629-632.

D. Kan, T. Terashima, R. Kanda, A. Masuno, K. Tanaka, S. Chu, H. Kan, A. Ishizumi, Y. Kanemitsu, Y. Shimakawa and M. Takano, Nat. Mater., 2005, 4, 816819.

E. Orhan, F. M. Pontes, C. D. Pinheiro, T. M. Boschi, E. R. Leite, P. S. Pizani, A. Beltrán, J. Andrés, J. A. Varela and E. Longo, J. Solid State Chem., 2004, 177, 38793885.

J. J. Yang, D. B. Strukov and D. R. Stewart, Nat. Nanotechnol., 2013, 8, 13-24.

J. Joshua Yang, M. X. Zhang, M. D. Pickett, F. Miao, J. Paul Strachan, W.-D. Li, W. Yi, D. A. A. Ohlberg, B. Joon Choi, W. Wu, J. H. Nickel, G. Medeiros-Ribeiro and R. Stanley Williams, Appl. Phys. Lett., 2012, 100, 113501.

K. H. Kim, S. Gaba, D. Wheeler, J. M. Cruz-Albrecht, T. Hussain, N. Srinivasa and W. Lu, Nano Lett., 2012, 12, 389-395.

W. Wen-Fa, C. Bi-Shiou and H. Shu-Ta, Semicond. Sci. Technol., 1994, 9, 1242.

D. Vaufrey, M. B. Khalifa, J. Tardy, C. Ghica, M. G. Blanchin, C. Sandu and J. A. Roger, Semicond. Sci. Technol., 2003, 18, 253.

Y.-F. Lan, Y.-H. Chen, J.-L. He and J.-T. Chang, Vacuum, 2014, 107, 56-61.

T. O. L. Sunde, E. Garskaite, B. Otter, H. E. Fossheim, R. Sæterli, R. Holmestad, M.-A. Einarsrud and T. Grande, J. Mater. Chem., 2012, 22, 15740.

S. Mochizuki, F. Fujishiro and S. Minami, J. Phys.: Condens. Matter, 2005, 17, 923-948. 
41. J. Joshua Yang, F. Miao, M. D. Pickett, D. A. Ohlberg D. R. Stewart, C. N. Lau and R. S. Williams, Nanotechnology, 2009, 20, 215201.

42. R. Waser and M. Aono, Nat. Mater., 2007, 6, 833-840.

43. H. Nili, T. Ahmed, S. Walia, R. Ramanathan, A. E. Kandjani, S. Rubanov, J. Kim, O. Kavehei, V. Bansal, M. Bhaskaran and S. Sriram, Nanotechnology, 2016, 27, 505210.

44. R. Waser, R. Dittmann, G. Staikov and K. Szot, Adv. Mater., 2009, 21, 2632-2663.

45. J. J. Yang, I. H. Inoue, T. Mikolajick and C. S. Hwang, MRS Bull., 2012, 37, 131-137.

46. Y. Meng Lu, M. Noman, Y. N. Picard, J. A. Bain, P. A. Salvador and M. Skowronski, J. Appl. Phys., 2013, 113, 163703.

47. J. H. Lee, E. J. Cha, Y. T. Kim, B. K. Chae, J. J. Kim, S. Y. Lee, H. S. Hwang and C. G. Park, Micron, 2015, 79, 101-109.

48. M. D. Pickett, J. Borghetti, J. J. Yang, G. MedeirosRibeiro and R. S. Williams, Adv. Mater., 2011, 23, 1730-1733.

49. K. D. Sung, Y. A. Park, H. R. Ryu, N. Hur and J. H. Jung, Solid State Commun., 2011, 151, 1784-1786.

50. F. Rossella, P. Galinetto, G. Samoggia, V. Trepakov and L. Jastrabik, Solid State Commun., 2007, 141, 9598.
51. K. Zhao, K.-j. Jin, Y. Huang, S. Zhao, H. Lu, M. He, Z. Chen, Y. Zhou and G. Yang, Appl. Phys. Lett., 2006, 89, 173507.

52. X. Z. Liu, B. W. Tao and Y. R. Li, J. Mater. Sci., 2007, 42, 389-392.

53. M. C. Ni, S. M. Guo, H. F. Tian, Y. G. Zhao and J. Q. Li, Appl. Phys. Lett., 2007, 91, 183502.

54. H. Tan, Z. Zhao, W.-b. Zhu, E. N. Coker, B. Li, M. Zheng, W. Yu, H. Fan and Z. Sun, ACS Appl. Mater. Interfaces, 2014, 6, 19184-19190.

55. A. Janotti, J. B. Varley, M. Choi and C. G. Van de Walle, Phys. Rev. B, 2014, 90.

A. Tebano, E. Fabbri, D. Pergolesi, G. Balestrino and E. Traversa, ACS Nano, 2012, 6, 1278-1283.

57. M. C. Tarun, F. A. Selim and M. D. McCluskey, Phys. Rev. Lett., 2013, 111, 187403.

58. D. S. Shang, J. R. Sun, L. Shi, Z. H. Wang and B. G. Shen, Appl. Phys. Lett., 2008, 93, 172119.

59. G. Bridoux, M. Villafuerte, J. M. Ferreyra, N. Bachi, C. A. Figueroa and S. P. Heluani, Phys. Rev. B, 2015, 92, 155202.

60. K. Zhao, Y. Huang, Q. Zhou, K.-J. Jin, H. Lu, M. He, B. Cheng, Y. Zhou, Z. Chen and G. Yang, Appl. Phys. Lett., 2005, 86, 221917.

61. J. Borghetti, V. Derycke, S. Lenfant, P. Chenevier, A. Filoramo, M. Goffman, D. Vuillaume and J. P. Bourgoin, Adv. Mater., 2006, 18, 2535-2540. 